\title{
A "new" approach to the quantitative statistica! dynamics of plasma turbulence: The optimum theory of rigorous bounds on steady-state transport
}

\author{
John A. Krommes
}

Princeton Plasma Physics Laboratory, P.O. Box 451, Princeton, NJ 0854s

Chang-Bae Kim

Institute for Fusion Studies, University of Texas at Awatin, Austin, Texas 78712

\begin{abstract}
The fundamental problem in the theory of turbulent transport is to find the flux $\Gamma$ of a quantity such as heat. Methods based on statistical closures are mired in conceptual controversies and practical difficulties. However, it is possible to bound $\Gamma$ by employing constraints derived rigorously from the equations of motion. Brief reviews of the general theory and its application to passive advection are given. Then, a detailed application is made to anomalous resistivity generated by self-consistent turbulence in a reversed-field pinch. A nonlinear variational principle for an upper bound on the turbulent electromotive force for fixed current is formulated from the magnetohydrodynamic equations in cylindrical geometry. Numerical solution of a case constrained solely by energy balance leads to a reasonable bound and nonlinear eigenfunctions that share intriguing features with experimental data: the dominant mode numbers appear to be correct, and field reversal is predicted at reasonable values of the pinch parameter. Although open questions remairi, upon considering all bounding calculations to date one can conclude, remarkably, that global energy balance constrains transport sufficiently so that bounds derived therefrom are not unreasonable and that bounding calculations are feasible even for involved practical problems. The potential of the method has hardly been tapped; it provides a fertile area for fut ure research.
\end{abstract}

\section{DISCLAIMER}

\begin{abstract}
This toport was prepared as an account of work sponsored by an agency of the United States Government. Neither the United States Government nor any agency thereof, nor any of their employess, makes any warranty, express or implied, or assumes any legal liability or iesponsibility for the accuracy, completeness, or usefulness of any information, apparatus, product, or process disclosed, or represents that its use would not infringe privately owned rights. Reference hercin to any specific commercial product, process, of service by trade name, trademark, mantfacturer, or otherwise does not necessarily constitute or imply ịts eadorsement, recommendation, or favoring by the United States Govcrnment of any ageincy :thereol. The, views and opinions of authors expressed berein do not necessarily state or reflect those of the United States Government or any agency thereol.
\end{abstract}

$$
\begin{aligned}
& \text { MASTER }
\end{aligned}
$$

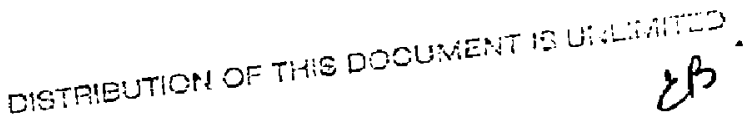




\section{INTRODUCTION}

In this article we discuss applications of the theory of rigorous bounds on transport ${ }^{1-5}$ due to steady-state turbulence. The method provides an intriguing alternative to the more familiar approaches based on statistical closure approximations. ${ }^{\circ}$ For a closely related but less mathematical discussion, see Ref. 7.

\section{A. The generic transport problem}

Consider for definiteness the prototypical dissipative nonlinear equation

$$
\frac{\partial}{\partial t} T(x, t)+\nabla \cdot[u(x, t) T]-\kappa \nabla^{2} T=0
$$

We distinguish two possibilities for the advecting field $u$, which for definiteness we assume to be solenoidal ( $\nabla \cdot u=0$ ). If $u$ is a functional of $T$, the problem is called "self-consistent"; otherwise, $u$ must be specified externally and the problem is called "passive." In both cases one assumes that $\mathrm{Eq}$. (1) exhibits statistical behavior, so it is relevant to introduce ensemble and/or spatial averaging operations. Statistical behavior may arise because of random initial conditions, intrinsic stochasticity, or, for passive advection, because $u$ is assumed by fiat to be a random variable.

We assume that the statistics are homogeneous in all directions but $x$. In the $x$ direction we assume that inhomogeneous, statistically sharp boundary conditions such as $T(x=0, t)=T_{0}, T(x=L, t)=T_{1}\left(T_{1} \neq T_{0}\right)$ are imposed such that a nont rivial steadystate profile $\langle T\rangle(x)$ is assured. Willow no sources on the right-hand side of Eq. (i). This precludes the possibility that $\partial\langle T\rangle / \partial x$ is constant; the fluctuations are intrinsically inhomogeneous, and the fundamental nonlinearity in the theory is the self-consistent adjustment of the profile to the fluctuations. Although extensions to homogeneous turbulence are possible, they are quite difficult and have not yet been developed: we shall not discuss them here.

For a complete statistical description of Eq. (1) one requires the joint probability distribution functional $P\{T, u, t\}$. However, besides being very difficult to obtain, $P$ provides an overabundanre of detailed information; one is generally content with much less. For example. the transport problem can be defined from the mean continuity equation:

$$
\frac{\partial}{\partial t}\langle T\rangle-\nabla \cdot \Gamma_{\mathrm{tor}}=0
$$

where we have assumed $\langle u\rangle=0$. The angular brackets denote a spatial average over the homogeneous directions (for self-consistent problems) or an ensemble average (for passive problems). The total flux $\boldsymbol{\Gamma}_{\text {tot }}$ is $x$-directed because of the stmmetry assumptions: $\boldsymbol{\Gamma}_{\mathrm{tot}}=$ 
$\hat{\boldsymbol{x}} \Gamma_{\text {tat }}$. Let us normalize $x$ to $L, u$ to $u_{\text {rms }} \doteq\left\langle\widetilde{u}^{2}\right\rangle^{1 / 2}$ [where $\left.\tilde{u} \doteq u-(u)\right]$, and $T$ to $\Delta T \doteq T_{0}-T_{1}$. Then $\Gamma_{\text {tot }}$ is the sum of a classical part $\Gamma_{\mathrm{el}}$ and an advective part $\Gamma$, where

$$
\begin{aligned}
\Gamma_{d} & \doteq-R^{-1} \frac{\partial(T)}{\partial x} \\
\Gamma & \doteq\left\langle\tilde{u}_{x} \tilde{T}\right)
\end{aligned}
$$

and $R \doteq u_{\mathrm{rm}} L / \kappa$ is a generic Reynolds-like number.

In steady state Eq. (1) reduces to $\partial \Gamma_{\text {tot }} / \partial x=0$, or $\Gamma_{\text {tot }}=$ constant. This constant is a functional of the fluctuations. To see this, observe that because of the special form (2a) it is useful to define the barring operation $\bar{A} \doteq \int_{0}^{1} d x A(x)$, so that $\bar{\Gamma}_{\mathrm{cl}}=-R^{-1} \int_{0}^{1} d x\langle T)^{\prime}(x)=$ $R^{-1}$ is entirely known in terms of the (statistically sharp) boundary conditions. Then, upon barring

$$
\Gamma_{\text {tot }}=\Gamma_{\mathrm{cl}}(x)+\Gamma(x)=\text { constant }
$$

we find

$$
\Gamma_{\text {tot }}=R^{-1}+\bar{\Gamma}
$$

in which the only unknown is $\bar{\Gamma}$. Determining $\bar{\Gamma}$ is the principal goal of a transport theory.

For future use, note that Eqs. (3) and (4) can be combined to express the mean profile in terms of the fluctuations:

$$
-\frac{\partial\langle T\rangle}{\partial x}=1-R \Delta \Gamma
$$

where $\Delta \Gamma(x) \doteq \Gamma(x)-\bar{\Gamma}$.

\section{B. Difficulties with conventional approaches}

One obvious way of determining $\bar{\Gamma}$ is to numerically integrate (many realizations of) Eq. (1), then to compute explicitly the ensemble and spatial averages. This procedure is nontrivial because of statistical noise and because the realizations need not be smooth. These difficulties are demonstrated explicitly in Ref. 8, where a one-dimensional version of Eq. (1) is studied in detail. A more subtle procedure is to determine analytically an approximate equation for $\Gamma(x)$, then solve that equation (probably numerically) and finally: spatially average the solution to compute $\bar{\Gamma}$. A variety of techniques exist for constructing such reduced descriptjons; their difficulties have been discussed exiensivel!: See Refs. 9 and 6 for reviews with many references. For a discussion oriented specifically to the transport problem, see Ref. 7. 


\section{The "optimum" variational method}

An alternative approach to the transport problem is suggested by the familiar observation that Eq. (1) determines an infinite hierarchy relating statistical moments of different orders. Thus, an infinity of constraints ${ }^{10}$ must be satisfied in order to uniquely determine $P\{T, u, t\}$ and, ultimately, the transport. Note, however, that most of the relations constrain extremely subtle details of $P$ and may be practically unimportant for the quantitative prediction of low-order moments such as $\bar{\Gamma}$. Optimistically, one could hope that just one or a small number of judiciously chosen constrajnts would suffice to predict $\bar{\Gamma}$ reasonably well.

For the specific problem of thermal convection, Malkus speculated ${ }^{11}$ that the steadystate flux was the maximum of all possible solutions of $\mathrm{Eq}$. (1) subject to the boundary conditions. Such a criterion is difficult to formulate analytically and furthermore turns out to be false except in certain limiting cases. However, it captures the right intuitive idea and suggests a possible way of proceeding. Indeed, by somewhat inverting the logic, Howard ${ }^{1}$ arrived at a nonlinear variational principle that was both rigorous and useful in practice. He posed the question "What is the maximum $\bar{\Gamma}$ subject to a finite subset of the infinity of constraints?" Let us denote such a bound by $\bar{\gamma}$. Of course, in the absence of any constraint at all the function space of all possible fields $\tilde{\boldsymbol{u}}(\boldsymbol{x})$ and $\tilde{T}(\boldsymbol{x})$ subject only to the boundary conditions is too vast; since such fields can be scaled arbitrarily, the unconstrained bound is infinity. However, Howard showed that one can demonstrate constrained variational problems that lead to finite $\bar{\gamma}$, and the rigorous nature of the formulation guarantees that $\bar{\gamma} \geq \bar{\Gamma}$.

In principle, further useful information would follow by bounding $\bar{\Gamma}$ from below as well as from above. Unfortunately, this problem is quite difficult. A lower bound for $\bar{\Gamma}$ is 0 , and any moment ronstraint is satisfied by the zero solution. Kraichnan has proposed sophisticated techniques to overcome this problem, ${ }^{12}$ but they remain to be fully developed. In the rest of this discussion we shall consider only bounds from above.

Conventionally; a fundamental constraint is derived from the steady-state balance of praduction $\mathcal{P}$, transfer $\boldsymbol{T}$, and dissipation $\mathcal{D}$. By definition, production is related to the interaction of the mean fields with the fluctuations, transfer describes conservative advection of the fluctuations, and dissipation is positive-definite when averaged over space. Although these quantities can be defined at various orders in the moment hierarchy: it is simplest and conventional to define them from the evolution equation of the variance of the fluctuations. One begins by multiplying the exact equation for the fluctuations;

$$
\frac{\partial}{\partial t} \tilde{T}(\boldsymbol{x}, t)+\nabla \cdot(\tilde{\boldsymbol{u}}\langle T\rangle-\tilde{\boldsymbol{u}} \tilde{T}-\langle\tilde{\boldsymbol{u}} \tilde{T}\rangle)-R^{-1} \Gamma^{2} \tilde{T}=0 .
$$

by $\tilde{T}(x, t)$ (at the same point in space and time); upon ensemble-averaging, one obtains

$$
\frac{\partial}{\partial t} \varepsilon(x, t)=P(x, t)+T(x, t)-D(x, t),
$$


where we have defined $\varepsilon \doteq\left\langle\tilde{T}^{2}\right\rangle / 2, \mathcal{P} \doteq-\Gamma(x) \partial_{x}\langle T\rangle, T \doteq-\partial_{x}\left\langle\tilde{u}_{z}\left(\tilde{T}^{2} / 2\right)\right\rangle$, and $\mathcal{D} \doteq$ $-R^{-1}\left\langle\tilde{T} \nabla^{2} \tilde{T}\right\rangle$. The troublesome term here is $T$, a triplet correlation function that de. scribes mode-mode coupling and advection of fluctuations from point to point. In statistical closure theory one attempts to approximate $T(x)$. By contrast, in the present method one appeals to the conservative nature of $T$ and the sharp boundary conditions to obtain rigorously $\bar{T}=0$. Therefore, by barring $\mathrm{Eq} .(6)$ one obtains in steady state the constraint that global dissipation balances global production:

$$
\overline{\mathcal{P}}=\overline{\mathbf{D}} \text {. }
$$

Krommes and Smith ${ }^{5}$ have called this the "basic" constraint. (It is often thought of as an energy balance, but $E$ need not be the physical fluctuation energy; it might, for example, be more closely related to entropy production.) Note that the unknown, spatially varying profile can be eliminated from $\mathcal{P}$ in favor of the fluctuations by using $\mathrm{Eq}$. (5), so

$$
\overline{\mathcal{P}}=\overline{\Gamma(1-R \Delta \Gamma)}=\bar{\Gamma}-R \overline{\Delta \Gamma^{2}}
$$

also, upon integrating by parts and using the boundary conditions one obtains the positivedefinite form

$$
\overline{\mathcal{D}}=R^{-1} \overline{\left\langle\left.\bar{\nabla} \bar{T}\right|^{2}\right\rangle}
$$

With the aid of Eqs. (8) and (9), one can rewrite Eq. (7) in the form

$$
\bar{\Gamma}=R^{-1} \overline{\left(|\nabla \tilde{T}|^{2}\right)}+R \overline{\Delta \Gamma^{2}}
$$

demonstrating that $\bar{\Gamma}$ is non-negative. This requirement is essential for successful use of the bounding method.

The simplest variational principle is, therefore, to maximize $\bar{\Gamma}$ subject to the basic constraint (7). If one employs the method of Lagrange multipliers, this amounts to maximizing the functional $\bar{\gamma}\{\tilde{T}, \overline{\boldsymbol{i}}\} \doteq \bar{\Gamma} \div \lambda(\bar{P}-\overline{\mathcal{D}})$. In this form the result is more general than its derivation from the specific equation (1).

One must inquire whether this singie constraint is sufficient. First, note that the cru. sial term in Eq. (10) is $\overline{\Delta \Gamma^{2}}$, which unlike the other two terms is of fourth order in the fluctuations. This is the fundamental nonlinearity mentioned above. It turns out that for selif-consistent problems the competition between second- and fourth-order terms is essential for obtaining a finite bound; when $(T)^{\prime}$ is taken to be constant $(=-1) . \Delta \Gamma$ vanishes and there is sufficient freedom that the bound is infinity. Kraichnan has pointed out ${ }^{12}$ that $f_{0 r}$ this case, which includes homogeneous turbulence. one must consider higher-order moments, but this is very difficult and has not yet been carried out. For passive problems. one can still obtain a finite bound $d^{5}$ for constant $\left\langle T^{\prime}\right\rangle^{\prime}$. but it is not very useful.

The basic constraint is not necessarily sufficient, even for inhomogeneous problems. In general, when $u$ is passive the basic constraint is enough to produce a well-posed variationa] 
problem with a finite bound. ${ }^{5}$ Self-consistent problems are more involved. Depending on the particular form of the dynamical equations and the chosen definition of $\boldsymbol{\varepsilon}$, often a second constraint is necessary in order to adequately pin down the relation between $\tilde{u}$ and $\tilde{T}$. However, in Sec. III we describe an example for which the basic constraint is sufficient.

Note that the basic theory produces time-independent Euler-Lagrange equations. This is a qualitative difference from the true physics. It means that the solutions of the EulerLagrange equations are at best suggestive of reality; however, one expects that they correctly capture aspects of the spatial dependence of an instantaneous snapshot of the true solution. One might find it remarkable that a time-independent theory can make rigorous predictions about equations that fluctuate randomly in time.

Since Howard's original work, the optimum approach has been applied to a variety of problems of interest in fluid dynamics; these were reviewed by Busse. All of the calculations described by Busse were seif-consistent and led to rather difficult problems in applied mathematics. (We shall see an example of such a calculation in Sec. III below.) However, the problem of passive advection is also of interest. It arises in a number of physical contexts, including the important problem of transport in specified stochastic magnetic fields, and it illustrates the method with a minimum of mathematical complexity. We shall briefly review results on the passive problem.

\section{BOUNDS FOR PASSIVE ADVECTION}

The general theory of bounds for passive advection was given by Krommes and $\mathrm{Smith}^{5}$ (KS). Although passive problems are in general simpler than self-consistent ones, in one sense they are more difficult: Whereas for self-consistent problems the autocorrelation time and/or length of $\tilde{u}$ can be computed self-consistently in terms of solely Reynolds' number, for passive problems the correlation function of $\tilde{\boldsymbol{u}}$ can be specified, thereby introducing extra parameters. As an example, KS considered a reference model a version of $\mathrm{Eq} .(1)$ in which the velocity field is taken to be a one-dimensional, centered Gaussian random variable $\tilde{u}(t)$ with (in sujtable dimensionless units) unit variance and autocorrelation time $K$ (Kubo number). This model can be solved exactly, either analytically ${ }^{5}$ for $K=\infty$ or numerically. ${ }^{*}$ For $K=\infty$, the basic bound $\bar{\gamma}_{\infty}$ is about $25 \%$ too high in the worst case $R \rightarrow \infty$. This is considered to be excellent agreement in view of the simplicjty of the calculation. (The direct-interaction approximation ieads to a somewhat more precise resuit. ${ }^{5}$ but at the cost of considerably more labor.) For finite $K$ the basic bound is qualitatively deficient: since it is constructed from equal-time and equal-space moments, it cannot contain information about the dynamical effects of finite correlation times and/or lengths related to the advecting velocity. Krommes and Snith pointed out that in this situation it was essential to invoke a two-time constraint: they implemented a simple one. Lpon solving an unusual integral equation, they found $\bar{\gamma}^{-1}=\bar{\gamma}_{a}^{-1}-\bar{\gamma}_{\infty}^{-1}$ : where $\bar{\gamma}_{a}$ is the quasilinear flux that is the true answer for $K \rightarrow 0$. This result is intuitively reasonable; it 
reduces naturally to both limits $K \rightarrow \infty$ and $K \rightarrow 0$. However, it must be stressed that this is much more than just a plausible interpolation formula; it is the rigonous bound on the fiux predicted by Eq. (1) (under the particular two-time constraint that was employed).

As a practical example, when the one-point basic bound is applied to the generic problem of particle transport in specified stochastic magnetic fields ${ }^{13}$ one obtwins ${ }^{5}$ the scaling correct for the strong turbulence regime. To do better and recover the familiar quasilinear result ${ }^{14, \mathrm{~L}^{3}}$ in the usual approximation of static fields one must employ a twospace-point constraint. Kim and Krommes have shown how to do this. ${ }^{8}$

\section{SELF-CONSISTENT BOUND FOR THE TURBULENT ELEC- TROMOTIVE FORCE IN REVERSED-FIELD PINCHES}

We would now like to gain experience with a self-consistent calculation relevant to plasma physies. As we have emphasized, the version of the bounding theory we describe here applies to problems in which the mean profile is imposed by inhornogeneous baundary conditions and is self-consistent with the fluctuations. This precludes its direct use for studies of turbulence in a region whose dimensions are small compared to macroscopic system size. ${ }^{5}$ For example, models of homogeneous turbulence with constant background gradient and periodic boundary conditions cannot be treated with the present method. Rather, what is needed is a macroscopic system with statistically sharp boundary conditions at the walls and describable uniformly in space by one "simple" set of partial differential equations. Such a model is furnished by the reversed-field pinch ${ }^{15}$ (RFP) in the resistive magnetohydrodynamic (MHD) description. This problem is of considerable theoretical and practical interest. Taylor's theory of relaxation in the presence of global helicity conservation ${ }^{16}$ has had considerable success in predicting features of the RFP such as field reversal. Montgomery and Phillips have explored related principles. ${ }^{17}$ However. it must be noted that none of these principles is rigorous, and they have little to say about the details of the underlying turbulence. The optimum theory offers intriguing possibilities for going beyond such work. First, it is entirely rigorous. Second, to the extent that the optimum variational principle reflects physical reality it makes definite staternents about the nonlinear fluctuations. Unfortunately; the extent to which this is true is difficult to predict a priori. so experience and, ultimately, much deeper insights are desirable. We shall now explore the predictions of the basic bound. However. since the calculation is quite involved we can only present the highlights here. An initial account of this work can be found in Ref. 18. For more details, see Ref. 19.

\section{A. The MHD description and turbulent electromotive force}

Consider a cylindrical pinch described by resistive, viscous. incompressible MHD. Ohm's law is

$$
\begin{gathered}
E_{\text {ext }}-E-c^{-j} u \times B=\eta j \\
-\tau-
\end{gathered}
$$


where $\boldsymbol{E}_{\text {ext }} \doteq \overline{\boldsymbol{z}} E_{0}$ is an external driving field and $\boldsymbol{E}$ is the internal field produced by the plasma. The resistivity $\eta$ is (unrealistically) assumed to be constant. We shall assume that $\boldsymbol{\nabla} \cdot \boldsymbol{u}=0$, so the mass density $\rho$ is constant. We also model the dissipative stress tensor as jsotropic, assuming the kinematic viscosity $\nu$ to be constant. The momentum equation is then

$$
\left(\frac{\partial}{\partial t}+u \cdot \nabla\right) u=-\nabla(P / \rho)+(\rho c)^{-1} j \times B+\nu \nabla^{2} u .
$$

(Although it has been argued that compressibility may be important for a precise de. scription of field reversal, ${ }^{20}$ reversal is still passible in the incompressible model, which leads to major analytic simplifications.) We consjder cylindrical geometry $(r, \theta, z)$ with constant toroidal field $B_{0}$. It is convenient to introduce as units of time, space, and magnetic field the resistive-diffusion time $\tau_{\eta} \doteq 4 \pi a^{2} / \pi c^{2}$, the minor radius $a$, and $B_{0}$. If we also introduce the viscous-diffusion time $\tau_{\nu} \doteq a^{2} / \nu$ and an Alfvén transit time $\tau_{A} \doteq a / c_{A}$, where $c_{A}^{2} \doteq B_{0}^{2} / 4 \pi \rho$, then it is natural to introduce as dimensionless parameters the magnetic Prandtl number $P_{m} \doteq \tau_{\eta} / \tau_{\nu}=4 \pi \nu / \eta c^{2}$ and the Hartmann number $H \doteq\left(\tau_{\eta} \tau_{\nu} / \tau_{A}^{2}\right)^{1 / 2}=\left(a^{2} B_{0}^{2} / c^{2} \eta \nu \rho\right)^{1 / 2}$. Then in terms of the vorticity $\omega \doteq \nabla \times u$ one can write Eg. (12a) as

$$
P_{\mathrm{m}}^{-1} \frac{\partial u}{\partial t}=-\nabla P^{\prime}+P_{m}^{-1} u \times \omega+H^{2} j \times B-\nabla \times \omega,
$$

where $P^{\prime} \doteq P / \rho+P_{\mathrm{m}}^{-1} u^{2} / 2$. We also have the (pre-)Maxwell equations

$$
\begin{aligned}
\nabla \times E & =-\frac{\partial B}{\partial t}, \\
\nabla \times B & =j \\
\nabla \cdot B & =0 .
\end{aligned}
$$

In addition to the assumption of steady state, we assume statistical homogeneity in the $\theta$ and $z$ directions. This, together with regularity at $r=0$, significantly constrains the form of the mean fields. For example. since $\partial(\ldots) / \partial \theta=\partial(\ldots) / \partial z=0$. Eq. $(13 c)$ reduces to $r^{-1} \partial\left(r\left\langle B_{r}\right\rangle\right) / \partial r=0$. the regular solution of which is $\left\langle B_{r}\right\rangle \equiv 0$. Similarly, we find $\left\langle u_{r}\right\rangle=\left\langle j_{r} .=\left\langle\omega_{r}\right\rangle=0\right.$. Since in steady state $\nabla \times\langle\boldsymbol{E}\rangle=0$ from Eq. (13a), we must have $\langle\boldsymbol{E}\rangle=-\nabla\langle\boldsymbol{\varphi}\rangle \propto \dot{\boldsymbol{r}}$. From these results we deduce that $\langle\boldsymbol{E}\rangle+\langle\boldsymbol{u}\rangle \times\langle\boldsymbol{B}\rangle \propto \dot{\boldsymbol{r}}$. Because of the presence of viscosity. we assume that $\langle\boldsymbol{u}\rangle$ vanishes at the wall.

As bouncary conditions on the fluctuations we shall assume that all fields are regular at $r=0$ and thai at the wall $\bar{u}=\widetilde{B}_{r}=r \times \widetilde{j}=0$. Other choices are possible and the issue is a difficult one. ${ }^{19.18}$ The present choice allows us to demonstrate the use of the optimum principle with a minimum of complications. 
We are now prepared to recognize the analogies to th generic transport problem sketched in Sec. I.A. Indeed, the curl of Eq. (11),

$$
\frac{\partial}{\partial t} B-\nabla \times(u \times B)-\eta \nabla^{2} B=0
$$

is analogous to Eq. (1). We shall assume that the total axial current is fixed and treat $E_{0}$ as a derived quantity. In this way one may treat the current as the driving force, analogous to $\Delta T$ in the geperic problem; $E_{0}$ is analogous to the total flux $\Gamma_{\text {tot }}$. More quantitatively, consider the mean Ohms' law

$$
\boldsymbol{E}_{\text {ext }}+\langle\boldsymbol{E}\rangle+\langle\boldsymbol{u}) \times\langle\boldsymbol{B}\rangle=\langle\boldsymbol{j}\rangle+\boldsymbol{Q}
$$

where $Q(r) \doteq\langle\tilde{B} \times \tilde{\mathbf{u}}\rangle(\boldsymbol{r})$. The $z$ component of Eq. (14) leads to

$$
E_{0}=\left\langle j_{s}\right)(r)+\varepsilon(r)
$$

where $\varepsilon(r) \doteq Q_{x}(r)[c f . \mathrm{Eq} .(2 b)\} ;$ Eq. (15) should be compared with Eqs. (3) and (2). In cylindrical geometry the natural barring operation is $\bar{A} \doteq 2 \int_{0}^{1} d r r . A(r)$. The driving force is then the mean axial current density $\mathcal{J} \doteq \overline{\left(j_{2}\right)}$. Upon barring Eq. (15), we obtain the alternative form of Ohm's law

$$
E_{0}=\mathcal{J}+\bar{\varepsilon}_{i}
$$

cf. Eq. (4). Upon combining Eqs. (15) and (16), we ubtain

$$
\left\langle j_{z}\right\rangle=\mathcal{J}-\Delta \varepsilon
$$

cf. Eq. (5). For future use, we can use this result and $\left\langle j_{r}\right\rangle=0$ to deduce

$$
\overline{\langle j\rangle \cdot Q}=J \bar{\varepsilon}-\left(\overline{\Delta \varepsilon^{2}}+\overline{Q_{\theta}^{2}}\right)
$$

\section{B. Energy constraint}

For the basic constraint we use the energy functional $\left(\left(\widetilde{u}^{2}+\widetilde{B}^{2}\right) / 2\right)$ and seek an upper bound on $\vec{E}$ constrained by global energ: balance. To determine the energ constraint we begin with the fluctuating parts of the curls of Eqs. (11) and (12):

$$
\begin{aligned}
& \frac{\partial}{\partial t} \tilde{B}=\nabla \times \tilde{u} \times(B)-(\boldsymbol{u}) \times \tilde{B}-(\tilde{B} \times \tilde{u}-Q)-\tilde{j} \\
& P_{m}^{-1} \frac{\partial}{\partial t} \tilde{u}=-\nabla \widetilde{P^{\prime}}-P_{m}^{-1} \tilde{u} \times(\omega)-(u) \times \tilde{w}-(\tilde{u} \times \tilde{w}-(\tilde{u} \times \tilde{w})): \\
& -H^{2}[\tilde{j} \times(B) \div(j) \times \tilde{B}+(\tilde{j} \times \tilde{B}-(\tilde{j} \times \tilde{B})) !-\nabla \times \tilde{w} \text {. } \\
& -9-
\end{aligned}
$$


Upon adding the scalar products of Eq. (18) with $\tilde{B}$ and Eq. (19) with $\tilde{\boldsymbol{u}}$ and barring the result, we are led to the steady-state condition

$\frac{\partial}{\partial t}\left[\frac{1}{\left(H^{2}\left(|\bar{B}|^{2}\right\rangle+P_{m}^{-1}\left\langle|\tilde{u}|^{2}\right\rangle\right)}\right]=0=C_{E} \doteq \overline{\langle u\rangle \cdot G}+H^{2} \overline{\langle j\rangle \cdot Q}-\left(\overline{H^{2}\left(\left.\bar{j}\right|^{2}\right\rangle}+\overline{\left\langle|\tilde{\omega}|^{2}\right\rangle}\right)$

where $G \doteq H^{2}\langle\tilde{B} \times \tilde{j}\rangle+P_{m}^{-1}\langle\tilde{\omega} \times \tilde{u}\rangle$. The mean of Eq. (12) can be used to show that (in steady state) $-\overline{\langle\boldsymbol{u}\rangle \cdot G}=\overline{|(\omega)|^{2}} \doteq 2 \Omega$. This result and Eq. (17) can then be used to demonstrate that $\bar{\varepsilon} \geq 0$, analogous to $\mathrm{Eq}$. (10). After considerable algebra an explicjt formula for $\boldsymbol{\Omega}$ in terms of the fluctuations can be obtained. ${ }^{10}$ Analogous manipulations lead one to a helicity balance constraint $C_{\mathrm{H}}$. Although we shall not deal with the helicity constraint in this work, it may be helpful to show in the equations to follow where the effects of that constraint would enter [terms of $\left.O\left(\zeta_{n}\right)\right]$. For more discussion, see Ref. 19.

\section{Variational principle}

We consider then the variational problem: Maximize

$$
\overline{\mathcal{A}}(\overline{\boldsymbol{u}}, \bar{B}) \doteq \bar{\varepsilon}+\lambda_{E} C_{E}+\lambda_{H} C_{H}+\overline{\lambda_{u}(x)(\nabla \cdot \tilde{u})}+\overline{\lambda_{B}(x)(\nabla \cdot \bar{B})}
$$

for fixed $\boldsymbol{J}$. After some algebra, the Euler-Lagrange equations can be cast into the form

$$
\begin{aligned}
& 0=\nabla \times \tilde{j}+\zeta_{\mathrm{h}} \tilde{j}-\tilde{u} \times L-H^{-2} \boldsymbol{\Omega}_{\mathbf{B}}+\nabla \hat{\lambda}_{\mathrm{B}}, \\
& 0=\boldsymbol{I}^{-2} \nabla \times \tilde{\omega}+\tilde{B} \times L-H^{-2} \boldsymbol{\Omega}_{u}+\nabla \bar{\lambda}_{u},
\end{aligned}
$$

where $\hat{\lambda} \propto \lambda$,

$$
\left.L \doteq\left[-Q_{\theta}+O\left(\zeta_{h}\right) ! \dot{\theta}-\frac{1}{2}\right) \zeta J-2 \Delta E+O \zeta_{h}\right): \dot{z}
$$

$\boldsymbol{\beta}_{\boldsymbol{v}} \doteq \delta \boldsymbol{\Omega} / \delta \boldsymbol{v}$ for $\boldsymbol{v} \in\{\tilde{\boldsymbol{B}}, \tilde{\boldsymbol{u}}\}, \zeta_{\mathrm{h}} \doteq \mathcal{J}\left(\lambda_{\mathrm{H}} / \lambda_{\mathrm{E}}\right)$ (in the absence of the helicity constraint, $\left.\zeta_{b} \equiv 0\right)$, and $\zeta \doteq 1+\lambda_{E}^{-1}+\mathcal{O}\left(\zeta_{h}\right)$. A relation between $\zeta$ and the fluctuations can be

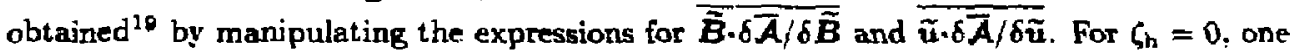
is evertually led to

$$
i=2-(\bar{\varepsilon} \mathcal{J})^{-1}\left[\overline{\left(\tilde{j}^{12}\right)}+H^{-2} \overline{\left(\bar{\omega} \tilde{\omega}^{12}\right)}\right] \text {. }
$$

It is convenient to eliminate $\lambda_{B}$ and $\lambda_{\psi}$ by applying the operators $\dot{r} \cdot(\nabla \times)^{n}(n=i$ or 2) to Eqs. (21). However, we roust still ensure that the associated solenoidal constraints $\boldsymbol{\nabla} \cdot \boldsymbol{B}$ and $\boldsymbol{\nabla} \cdot \boldsymbol{u}$ remain satisfied. Therefore. we adopt the representation

$$
\tilde{B}=\nabla \times(\dot{r} ; B)-\nabla \times \nabla \times(\dot{r} \times B)
$$


and similarly for $\tilde{u}$. (Fo: $\psi$ and $\chi$, the subscripis $\boldsymbol{B}$ or $u$ ase just labels; they do not denote differentiation.) We decompose $\boldsymbol{L}$ into lirenar and nonlinear parts, $\boldsymbol{L}=\boldsymbol{L}_{\mathrm{j}}+\boldsymbol{L}_{\mathrm{n}}$, where

$$
\begin{aligned}
& L_{1} \doteq \frac{1}{2} T \zeta \dot{x}+D\left(\zeta_{h}\right) \hat{\theta} \\
& \boldsymbol{L}_{\mathrm{a}} \doteq\left[-Q_{+}+\boldsymbol{O}\left(\zeta_{b}\right)\right] \hat{\boldsymbol{\theta}}+\left[-\Delta \varepsilon \nrightarrow \boldsymbol{O}\left(\zeta_{h}\right)\right] \hat{z} \text {. }
\end{aligned}
$$

Then we ohzain tide conärses, time-independent boundary-value problem

$$
\begin{aligned}
& \hat{f} \nabla \times \nabla \times \bar{j}+\zeta_{h}+\nabla \times \bar{j}-L_{j} \cdot \nabla \tilde{u}_{r}=C_{w e}, \\
& H^{-2} \dot{r} \cdot \nabla \times \nabla \times \tilde{\omega}+L_{1} \cdot \nabla \bar{B}_{r}=C_{\nabla_{-1}}
\end{aligned}
$$

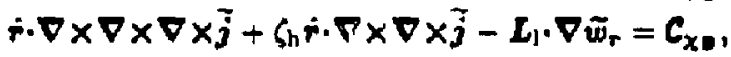

$$
\begin{aligned}
& H^{-2} \dot{r} \cdot \nabla \times \nabla \times \nabla \times \tilde{w}+L_{l} \cdot \nabla \bar{j}_{r}=C_{x_{x}}
\end{aligned}
$$

where

$$
\begin{aligned}
& C_{\psi_{n}} \doteq L_{\mathrm{n}} \cdot \nabla \tilde{u}_{r}-H^{-2} \hat{\mathrm{p}} \cdot \nabla \times \Omega_{B}, \\
& c_{\psi_{n}} \doteq-L_{n} \cdot \nabla \tilde{B}_{\mathrm{T}}-H^{-2}+\nabla \times \Omega_{u} \\
& \tau_{x=} \doteq \dot{p} \nabla \times \nabla \times\left(\tilde{u} \times L_{n}\right) \cdots H^{-2} \dot{r} \nabla \times \nabla \times \Omega_{B} \text {, } \\
& c_{x_{u}} \doteq-+\nabla \times \nabla \times\left(\tilde{B} \times L_{n}\right) \cdots H^{-2}+\nabla \times \nabla \times \Omega_{u} \text {. }
\end{aligned}
$$

The highest order of the derivatives in each of Eqs. (22c) and (22d) is 6 [since, e.g., $j=\nabla \times B \sim \nabla \times \nabla \times \nabla \times(\hat{r} \times B)] ;$ therefore; the system is of twelfth order. Determining the explicit expressions of the various curls requires tedieus algebra; the results may be found in Ref. 19.

\section{Energu stability and the critical current}

The solution of th: Euler-Lagrange prablem determines the bounding curve $\bar{E}(\mathcal{J})$ or, equivalently, $J(\bar{\varepsilon})$. The latter form is often more convenient because the point $\bar{\varepsilon}=0$ has a special status. Namely, since $\vec{\varepsilon} \geq 0$, the quantity $\mathcal{J}_{c} \doteq J(0)$ determines the critical current bejow which no solution exists to the Euler-Lagrange equations. It can be shown ${ }^{10}$ that $J_{c}$ is identical io the entergy stability criterion ${ }^{3,21}$ : for currents below $\mathcal{J}_{\varepsilon}$. perturbations of arbitrary size decay monotonirally, so finite-amplitude steady-state turbulence cannot exist for $\mathcal{J}<\mathcal{J}_{c}$. Since energ: stability is a very' strong requirement. it is not surprising that $\mathcal{J}_{s}$ is very small. Nevertheless, because $\mathcal{J}_{c}$ is the first point on the bounding curve, it is important to compute it: The shape of the critical eigenfunctions provides the natural first guess for iterative numerical solution for sl.sal] but nnite $\bar{\varepsilon}$.

For $\bar{\varepsilon}=0$ the right-hand sides of Eqs. (22) vanish and we are led to a linear eigenvalue problem. To solve this, we first Fourier transiorm in the homogeneous directions $\theta$ and $z$ : $\tau=\sum_{k} \varphi_{h}\left(r, \operatorname{xpp}\left(i\left(m \theta+k_{z} z\right)\right)\right.$ where $\psi \in\{r \cdot \lambda\}, k \doteq\left(m, k_{z}\right)$, and $k_{z} \doteq 2 \pi n / L$. The 
operator $\Lambda_{k} \doteq-i L_{1} \cdot \nabla=k_{z} J / 2+O\left(\zeta_{k}\right)$ playss the role of eigenvalue for fixed $k$. From $\Lambda_{k}$ we then obtain $\mathcal{J}_{\boldsymbol{h}}$; the ciesired critical current is $\mathcal{J}_{c}=\inf _{\boldsymbol{h}} \mathcal{J}_{\boldsymbol{k}}$.

For $\zeta_{h}=0$ the Hartmann rumber can be removed by the rescaling $\tilde{\boldsymbol{B}}=\boldsymbol{H}^{-1} \tilde{\boldsymbol{B}}^{\prime}$, $\Lambda_{h}=H^{-1} \Lambda_{h}^{\prime}$. The resulting system is somewhat analogous to Bessel's equation and must be solved numerically. Looking ahead to the nonlinear problem we choose the procedure of Lentini and Pereyra, ${ }^{22}$ a variable-order, variable-step-size finite-difference method with deferred corrections. ${ }^{23}$ To ensure that the variables remain regular at the origin, we follow the analysis of Lentini and Keller, ${ }^{24}$ Keller, ${ }^{25}$ and de Hoog and Weiss. ${ }^{20}$ In this procedure the system is written in the form $y^{\prime}=r^{-1} A(r) y+B(r, y) y$, where $A$ and $B$ are regular as $r \rightarrow 0$. To determine the behavior near the origin we may ignore $B$; then, in the special case that $A$ can be diagonalized the variables $z$ in the diagonal representation obey $z_{i} \propto r^{\Lambda_{2}}$, where $\Lambda_{i}$ is an eigenvalue of $A$. Regularity requires that all $z$ 's with negative $\Lambda$ be set to 0 . These conditjons constrain linear combinations of the $y$ 's to vanish. In practice, we apply these conditions at $r=\epsilon$, where $\epsilon \ll 1$. In the present problem $A$ cannot be diagonalized for most $m$ 's; however, it can be brought to Jordan canonical form and an analogous procedure applied. The details are quite involved. ${ }^{19}$

The results of the critical current calculation are ${ }^{19}$ that the maximizing modes are $m= \pm 1, n= \pm 2$ for unit aspect ratio $A$, and that $J_{c} \approx 40 \mathrm{H}^{-1}$. (To date, we have studied only $A=1$, which is an interesting exemplary case.) Since $H$ may be very large ! $H \sim(\pi \nu)^{-1 / 2}$, , the critical current is far below the actual value observed in the RFP experiments $j=O(1)$ in the present units. However, the dominance of $m=1$ modes is in agreement with both numerical simulations and experiments. Also, it is in accord with the speculation of Caramana ${ }^{27}$ that $|n| \sim 2 R / a$.

\section{E. Single-mode bounding curve}

We nox tum to the solution of the nonlinear system (22). Again, for arbitrary $H$ this must be done numerically. For very large $H$ (as in many experiments), an analytic calculation using singular perturbation theory is suggested ${ }^{4}$; however, this has not been attempted yet.

It is characteristic that the Euler-Lagrange equations derived from the optimum princjple have nonlinearit ues of speciaj form. ${ }^{4}$ Specifically, the coefficients of $L_{n}$ depend only on $\boldsymbol{r}$, as do the coefficierits of $\tilde{\boldsymbol{u}}$ or $\tilde{\boldsymbol{B}}$ in $\boldsymbol{\Omega}_{\mathrm{t}}$ or $\boldsymbol{\Omega}_{\boldsymbol{B}}$. Thus. Fourjer transformation in $\theta$ and $z$ is still appropriate. $V$ alike the linear problem. however. the Fourier modes are now coupled through the values of the nonlinear terms, which are determined by sums over al] modes, and it is not guaranteed that solutions with single $k$ maximize $\bar{\xi}$. Presumably such solutions are appropriate for $\bar{\xi}$ sufficiently close to 0 ( $\mathcal{J}$ close to $\mathcal{J}_{c}$ ). (This does not necessarily imply that pert urbation theory is adequate.) Motivated by experience with simpler problems. ${ }^{4}$ it is presumed that as $\mathcal{J}$ is increased bifurcations eventually occur such that at $\mathcal{J}=\mathcal{J}_{n} n$-mode solutions replace $(n-1)$-mode solutions as the maximizing ones. For our 
initial analysis of this extremely complicated problem we shall consider only single-mode solutions. In particular, we retain only the $(m, n)=(1,-2)$ mode, for which the rational surface falls inside the plasma. Furtbermore, for reasons of computational simplicity we shall neglect in our preliminary calculations the terms describing the effects of the mean velocity. The resulting system is undetermined in the sense that $R e B_{h}=C_{k} \ln B_{k}$ for arbitrary real $C_{k}$. However, for the single-mode solution it can be shown that $\bar{\varepsilon}$ is independent of $C_{k}$. It is expected that this approximation affects the final bound by an amount of $O(1)$.

In the absence of the helicity constraint the bounding curve satiafies the similarity law $H \mathcal{J}=f(H \bar{\varepsilon})$. The function $f$ has been determined numerically for $H \mathcal{J} \lesssim 2 \times 10^{3}$; see Ref. 18 for a graph. At the largest values of $H \bar{\varepsilon}$ boundary layers are evident; this is consistent with the observation that the bounding curve appears to have tatered its asymptotic regime, being essentially linear on a $\log -\log$ plot for $H \mathcal{J} \geq 5 \times 10^{2}$. In that regime we obtain

$$
\bar{E} \approx 0.4 H^{0.1} \mathcal{J}^{1.1} \text {. }
$$

Near the critical point $\bar{\varepsilon} \propto J-J_{c}$ as can be demonstreted analytically.

Examples of the nonlinear eigenfunctions are displayed in Ref. 19. The optimum profiles display field reversal for sufficiently large currents ${ }^{18}$; extrapolating a few data points obtained at modest $\boldsymbol{H}$ leads to a prediction of field reversal at a pinch parameter of $\approx \mathbf{1 . 5}$ for $H \sim 10^{5}$, which is quite reasonable. The optimum states are not Taylor-like both near the wall (as would be expected) and near the center.

Further work must be done to explore in more detail the predictions and properties of the optimum states and to understand their relation to physical reality. However, we conclude that the energy upper bound on the turbulent emf is not unreasonable. This is in accord with previous experience ${ }^{4}$ with self-consistent fluid problems. An abvious but technically quite difficult ${ }^{19}$ extension of this caiculation would be to include the helicity constraint.

\section{DISCUSSION}

In summary: the theory of bounds is a "new" appraach to the theory of plasma turbulence: though its history spans more than thirty years, to our knowledge the first discussion of it in the plasma physics literature is given in Ref. 21. Our calculations merely scratch the surface of possible plasma applications. Furthermore, there is renewed interest in the lounding technique in general because of $j$ ts possible marriage with other approaches such as constrained Jecimation. ${ }^{28,29}$ Although space does not permit a description of the techniques that have been proposed ${ }^{12}$ for obtaining nontrivial lower bounds and extending the method to homogeneous turbulence, it is clear that such calculations would be highly. desirable. In general, the theory of bounds on tarbulent transport presents a fertile and challenging area for further research. 


\section{ACKNOWLEDGEMENTS}

This work was supported by U.S. Department of Energy Contract Nos. DE-AC0276CHO3073 and DE-FG05-80ET53088. We are grateful for many detailed discussion with Dr. Ralph Smith, and acknowledge the inspiration of Ref. 21. Finally, we thank both Dr. Maurizio Ottaviani and an anonymous referee, who read the manuscript with great care and offered a variety of suggestions that significantly improved the final version.

\section{REFERENCES}

1 L. N. Howard, J. Fluid Mech. 17, 405 (1963).

2 L. N. Howard, Ann. Rev. Fluid Mech. 4, 473 (1972).

S D. D. Joseph, Stability of Fluid Motions (Springer-Verlag, New York, 1976).

4 F. H. Busse, Adv. Appl. Mech. 18, 77 (1978), and references therein.

5 J. A. Krommes and R. A. Smith, Ann. Phys. 177, 246 (1987).

6 J. A. Krommes, in Basic Plasma Physics II, edited by A. A. Galeev asd R N. Sudan (North-Holland, Amsterdam, 1984), Chap. 5.5.

7 J. A. Krommes, in International Conference on Plasma Physics (Pramãna, Indian Academy of Sciences, Bangalore, India, in press).

C.-B. Kim and J. A. Krommes, J. Stat. Phys. 53, 1103 (1988).

${ }^{9}$ R. H. Kraichnan, J. Stat. Phvs. 51, 949 (1988).

${ }^{10}$ R. H. Kraichnan, in Nonlinear Dynamics, edited by H.G. Helleman (New York Academy of Sciences, New York, 1980), p. 37.

${ }^{11}$ W. Malkus, Proc. Roy: Soc. A 225, 196 (1954).

12 R. H. Kraichnan, in Institute for Fusion Studies Report No. IFSR 318 (1987).

13 J. A. Krommes, C. Oberman, and R. H. Kleva, J. PJasma Phirs, 30, 11 (1983).

${ }^{14}$ A. B. Rechester and M. N. Rosenbluth, Phvs. Rev. Lett. 40, 38 (I978).

15 H. A. Bodin and A. A. Newton, Nucl. Fusion 20, $125 \overline{5}$ (1980). 
16 J. B. Taylor, Rev. Mod. Phys. 58, 741 (1986).

17 D. Montgomery and L. Phillips, Phys. Rev. A 38, 2953 (1988).

18 C.-B. Kim and J. A. Krommes, submitted to Phys. Rev. Lett. (1989).

${ }^{19}$ C.-B. Kim, Ph.D. thesis, Princeton University, 1989.

${ }^{20}$ D. Y. Aydemir, D. C. Barnes, E. J. Caramana, A. A. Mirin, R. A. Nebel, D. D. Schnack, and A. G. Sgro, Phys. Fluids 28, 898 (1985).

22 R. A. Smith, Ph.D. thesis, Princeton University, 1986.

22 V. Pereyra, in Lecture Notes in Computer Science (Springer-Verlag, Berlin, 1978), Vol. 76, p. 67.

29 This algorithm is implemented in the International Mathematical Subroutine Library (IMSL) [see, e.g., Sources and Development of Mathematical Software, edited by Wayne R. Cowel! (Prentice-Hall, Englewood Clifts, NJ, 1984)] as the routine DVCPR.

24 M. Lentini and H. B. Keller, SLAM J. Numer. Anal. 17, 577 (1980).

25 H. B. Keller, Numerical Solution of Two Point Boundary Value Problems (SIAM, Philadelphia, 1976).

${ }^{26}$ F. R. de Hoog and R. Weiss, SIAM J. Numer. Anal. 13, 775 (1976).

27 E. J. Caramana, Phys. Fluids B 1, 2186 (1989).

$28 \mathrm{R}$. H. Krajchnan, in Theoretical Approaches to Turbujence, edited by D. L. Dwoyer, M. Y. Hussaini, and R. G. Voight (Springer. New York, 1985), p. 91.

29 R. H. Kraichnan and S. Chen, Physica D 37, 160 (1989). 